\title{
Poincaré, creador de los métodos todavía modernos en las ecuaciones diferenciales y en la mecánica celeste
}

\author{
Amadeu Delshams i Valdés
}

Arbor CLXXVIII, 704 (Agosto 2004), 669-689 pp.

Con motivo del sesquicentenario del nacimiento de Henri Poincaré, resulta impresionante comprobar la influencia actual de su obra, así como el gran adelanto de sus métodos e ideas respecto a las de los científicos coetáneos. En esta conferencia se repasan algunas de sus contribuciones principales a las ecuaciones diferenciales y a la mecánica celeste, y se discute el papel central que tuvo en éstas su memoria sobre el problema de tres cuerpos presentada en 1889 a un concurso para conmemorar el sexagésimo aniversario del Rey Óscar II de Suecia y Noruega.

En particular, el descubrimiento de un error esencial en la memoria presentada por Poincaré le llevó al descubrimiento de las órbitas homoclínicas, que son la causa principal de generación de movimiento caótico, con lo que Poincaré se avanzó en muchos años a los descubridores posteriores de movimiento complicado en los sistemas dinámicos.

\section{Introducción}

Cuando se cumple el sesquicentenario de Poincaré, resulta impresionante volver la vista atrás, y reconocer el gran impulso que las ecuaciones diferenciales y la mecánica celeste recibieron de él. Además, es muy 
claro comprobar el hecho de que Poincaré se avanzó en mucho a sus coetáneos, en tanto y cuanto introdujo una gran variedad de métodos totalmente nuevos, combinando métodos cuantitativos con cualitativos, con topológicos y geométricos.

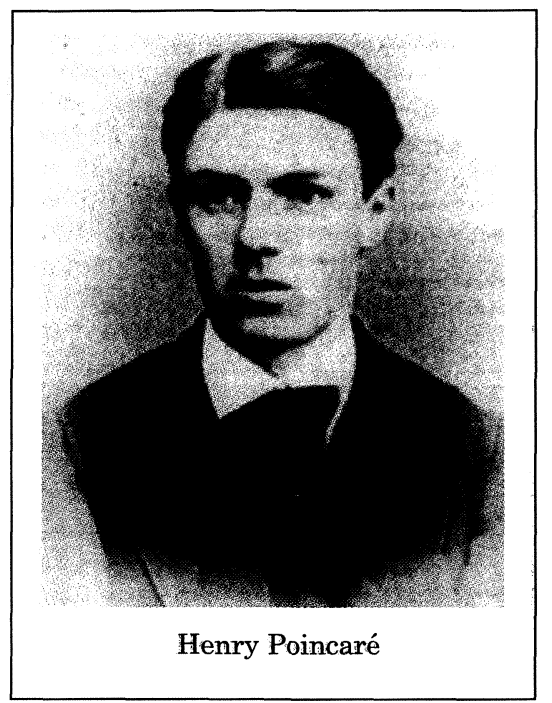

En esta conferencia vamos a ver que un hecho central en su carrera, al menos en lo referente a las ecuaciones diferenciales y a la mecánica celeste, fue la presentación en 1889 de una memoria sobre el problema restringido de tres cuerpos al concurso para conmemorar el sexagésimo aniversario del Rey Óscar II de Suecia y Noruega. Sus resultados anteriores a esta fecha le fueron de gran importancia para el desarrollo de la memoria, pero el esfuerzo realizado por Poincaré durante los años 1887 a 1889 fue extraordinario, más aún si se tiene en cuenta que por en medio tuvo que arreglar un error esencial cometido en la memoria, que invalidaba su resultado sobre la estabilidad del problema restringido de tres cuerpos. Sin embargo, al corregir este error, y en poco menos de medio año, Poincaré fue capaz de descubrir las órbitas homoclínicas, cuya existencia es la causa principal de existencia de movimiento caótico en un sistema. En este sentido se avanzó de manera considerable a los conocimientos existentes en su tiempo, hasta el punto que aún hoy en día los trabajos de Poincaré, especialmente los relacionados con su memoria, son fuente de inspiración para trabajos innovadores, con lo que se pone de manifiesto la vigencia actual de su obra.

\section{Antes de 1889}

En su tesis Sur les propriétés des fonctions définies par les équations aux différences partielles [27], publicada en 1879, Poincaré estudió la linealización analítica de un campo vectorial en un entorno de un punto de equilibrio, a través de la existencia de soluciones analíticas de ecuaciones en derivadas parciales casi lineales de primer orden. En particular, descubrió que cuando los exponentes característicos del punto de equilibrio 
Poincaré, creador de los métodos todavía modernos...

de un campo vectorial analítico de dimensión arbitraria cumplían una condición algebraica, la así llamada condición de no resonancia, junto con una condición geométrica, consistente que el origen no esté contenido en la envolvente convexa en el plano complejo de los exponentes característicos, entonces existe un cambio analítico en un entorno del punto de equilibrio que lo transforma simplemente a su parte lineal.

Actualmente, esta condición geométrica es conocida como dominio de Poincaré (véase, por ejemplo, [4], y se ha revelado como uno de los pocos casos para los cuales se puede garantizar la linealización analítica de un campo vectorial. Es una muestra inequívoca de su genio la elegancia con la que utiliza el método de los mayorantes, introducido por Cauchy con el nombre de cálculo de los límites dentro de los cuales las series formales obtenidas eran convergentes.

Pero es a continuación de su tesis cuando Poincaré engendra lo que se conoce hoy en día como teoría cualitativa de ecuaciones diferenciales ordinarias, a través de su Memoria sobre las curvas definidas por una ecuación diferencial [28, 29, 32, 33], que de hecho consta de cuatro partes repartidas entre 1881 y 1886 .

En estos artículos, Poincaré, consciente de la imposibilidad de integrar la vasta mayoría de ecuaciones diferenciales ordinarias mediante cuadraturas de funciones conocidas, se propone realizar un estudio geométrico que combine el estudio local de las soluciones con el estudio global, haciendo intervenir, cuando haga falta, los métodos topológicos que él mismo desarrolla.

Ya al introducir este tipo de estudio cualitativo en el primero de los artículos de esta memoria, Poincaré se plantea su posterior aplicación en los problemas de mecánica celeste, entre los cuales cita en particular el problema de los tres cuerpos, sobre el cual formula diversas cuestiones, principalmente relacionadas con la estabilidad de las trayectorias.

Sin embargo, en las dos primeras partes de la memoria sobre las curvas definidas por una ecuación diferencial, Poincaré limita su estudio a las ecuaciones diferenciales de dimensión dos. En su estudio local de los puntos de equilibrio, Poincaré introduce su índice que permite, por ejemplo, diferenciar a los puntos silla de los nodos, que después dio lugar al teorema del índice de Poincaré para superficies cerradas. En particular, comprueba que todo campo sobre la esfera contiene puntos de equilibrio.

También introdujo las líneas de sección y sus aplicaciones de retorno asociadas, que actualmente se conocen como secciones de Poincaré y aplicaciones de Poincaré, respectivamente, y utilizó estas herramientas para determinar la existencia de ciclos límite, es decir, soluciones periódicas aisladas, así como sobre su posible distribución. 
Las dos primeras partes de la memoria aparecieron casi sucesivamente, mientras que las dos últimas no lo hicieron hasta al cabo de de tres años. En la introducción de la tercera parte, Poincaré pone de manifiesto la semejanza entre las cuestiones tratadas en las dos primeras partes de la memoria y el problema de estabilidad del sistema solar, mucho más difícil, al ser necesario considerar sistemas de orden mucho mayor a dos, y se plantea, así, estudiar sistemas de dimensión más alta, al menos al final de la memoria.

En las dos últimas partes de esta memoria, Poincaré estudia los campos vectoriales sobre superficies, generalizando su teorema del índice para superficies diferentes a la esfera. Esto le lleva a estudiar campos vectoriales sobre el toro, donde se encuentra con problemas de pequeños denominadores, asociados a números de rotación irracionales, y similares a los que se encontrará más adelante al considerar las aproximaciones del método de Lindstedt usadas en mecánica celeste, así como con la existencia de campos vectoriales sin puntos de equilibrio.

Trabajando con campos vectoriales multidimensionales, Poincaré se da cuenta que puede utilizar también las hipersuperficies de sección y las aplicaciones de Poincaré, de manera que el estudio del comportamiento alrededor de una órbita periódica se reduce al de los iterados de una aplicación en un entorno de un punto fijo. E introduce los exponentes característicos asociados a una órbita periódica, los cuales le proporcionan, en primer orden, la información sobre su estabilidad, así como los invariantes integrales, que más tarde serán de una gran importancia para el caso de sistemas hamiltonianos. Se encuentra de nuevo con los pequeños divisores al intentar calcular las series de las posibles integrales primeras o de las soluciones del sistema.

De hecho, Poincaré ya había trabajado con series trigonométricas en $[31,34]$, insistiendo entre la diferencia entre la convergencia puntual y la convergencia uniforme, así como las condiciones sobre los coeficientes de Fourier que permiten derivar término a término las series de Fourier, siempre pensando en su aplicación a las series provenientes de mecánica celeste.

Finalmente, Poincaré había ya abordado en 1884 el estudio de órbitas periódicas del problema de tres cuerpos [], y generalizó el trabajo de Hill, probando la existencia de un continuo de órbitas periódicas del problema de tres cuerpos, que después clasificó en tres tipos, en función de su excentricidad e inclinación. Estos resultados le fueron de de gran importancia para la posterior discusión de órbitas periódicas que llevó a cabo en [36]. 
Poincaré, creador de los métodos todavía modernos...

\section{La memoria sobre el problema de tres cuerpos}

En el año 1 884, Gösta Mittag Leffler, profesor de matemáticas puras de la Universidad de Estocolmo, le propone al Rey Óscar II de Suecia y Noruega la realización de un concurso matemático con el fin de conmemorar el sexagésimo aniversario del monarca, que tendría lugar cinco años más tarde, más concretamente el 21 de enero de 1889.

Este tipo de concursos no eran inusuales durante el siglo XIX y, aunque los premios ofrecidos no eran económicamente muy altos, proporcionaban un prestigio considerable a los ganadores. Además, Mittag-Leffler había fundado la revista matemática Acta Mathematica dos años antes y, como su editor jefe, quería asociar este concurso a la revista, para darle más publicidad y prestigio a la revista, esperando que atrajese artículos importantes.

El Rey Óscar, que sentía una debilidad manifiesta por las matemáticas, como consecuencia de haber cursado algunas asignaturas de matemáticas en la Universidad de Uppsala, ya había ayudado anteriormente a Mittag-Leffler en la fundación de Acta Mathematica y aceptó rápidamente la idea de Mittag-Leffler, ofreciendo un premio consistente en una medalla de oro y 2500 coronas. Aunque el valor económico del premio no era muy alto (para apreciarlo mejor, digamos que el sueldo anual de Mittag-Leffler de 7000 coronas por entonces), el hecho de que estuviese patrocinado por todo un Rey de Suecia le daba un prestigio comparable al que podían tener, por ejemplo, los futuros premios Nobel.

El diseño del jurado no fue fácil. Por un lado, la ausencia de cualquier matemático distinguido restaba prestigio al premio y alimentaba las quejas del campo o país correspondiente sobre posibles partidismos. Por otro lado, debido al fuerte carácter y rivalidades existentes entre algunos matemáticos importantes, por el bien del premio había que evitar poner en el tribunal a matemáticos claramente enfrentados. Así pues, Mittag-Leffler se decidió por un tribunal muy próximo a él, compuesto por sus antiguos mentores Hermite y Weierstrass, como representantes de la escuela francesa y alemana, y él mismo, como editor jefe de Acta.

La decisión del tema o problemas del concurso también llevó su tiempo. Por un lado, dejar el tema totalmente abierto conllevaba el problema de tener que comparar trabajos sobre materias muy dispersas y desconocidas para los miembros del tribunal, y la resolución del premio abriría así las puertas a quejas sobre desconocimiento o partidismo del tribunal. Por otro lado, limitarlo a un único problema cerraba la puerta a muchos posibles concursantes, con lo que la calidad del premio se podía ver afec- 
tada. Finalmente se optó por plantear cuatro preguntas, dejando abierta la posibilidad para que se presentasen trabajos que versasen sobre otros temas, en el bien entendido de que se daría prioridad a las memorias que tratasen las cuatro preguntas planteadas.

El anuncio oficial del concurso se publicó a mediados del año 1885 en Acta y en Nature. En el anuncio se mencionaba el premio, se nombraba la comisión, se planteaban las cuestiones y se estipulaban las condiciones de entrega de las memorias, las cuales tenían que estar en manos del editor jefe de Acta antes del uno de junio de 1888, con lo que el plazo era de tres años.

De las cuatro preguntas, Weierstrass formuló una y Hermite tres. La primera, que versaba sobre el problema de $n$ cuerpos y había sido planteada por Weierstrass, parecía la más importante, al menos teniendo en cuenta su redacción y la longitud y detalles de su formulación:

Dado un sistema formado por un número arbitrario de puntos materiales que se atraen mutuamente de acuerdo con las leyes de Newton, se propone, bajo la hipótesis de que un choque entre dos o más partículas no tiene nunca lugar, desarrollar las coordenadas de cada partícula en una serie procedente de funciones conocidas del tiempo y que sean uniformemente convergentes para cualquier valor del tiempo.

Parece ser que este problema, cuya solución ampliará nuestro conocimiento sobre el sistema del universo, puede ser resuelto por medio de las herramientas analíticas de que se dispone actualmente; esto es al menos lo que cabe suponer, ya que poco antes de su muerte Lejeune-Dirichlet comunicó a un amigo suyo ${ }^{1}$, matemático, que había descubierto un método para integrar las ecuaciones diferenciales de la mecánica, y que él había tenido éxito, al aplicar este método, en demostrar la estabilidad de nuestro sistema planetario de manera totalmente rigurosa. Desafortunadamente no sabemos nada sobre este método, excepto que el punto de partida para este descubrimiento parece haber sido la teoría de las oscilaciones infinitesimales. Sin embargo, se puede suponer casi con total certeza que este método no estaba basado en largos y complicados cálculos, sino en el desarrollo de una idea fundamental simple, que se espera razonablemente que se pueda encontrar de nuevo por medio de un estudio más serio y perseverante.

Sin embargo, en caso de que nadie tenga éxito en resolver el problema propuesto dentro del plazo del concurso, el precio podría ser otorgado a un trabajo en el cual algún otro problema de la mecánica sea tratado en la forma indicada y sea resuelto completamente.

1 Leopold Kronecker 
Poincaré, creador de los métodos todavía modernos...

Del enunciado de esta cuestión se desprende tanto el gran interés que tenía Weierstrass por este problema, que había intentado atacar juntamente con Sofía Kovalevskaya, como el gran respeto que le merecía la reputación de Dirichlet, cuyas afirmaciones le inducían a creer que se podía probar la convergencia de algún desarrollo para las soluciones del problema de tres cuerpos.

De las tres preguntes restantes, dos fueron formuladas por Weierstrass, y la última por Hermite. Así, la segunda de las preguntas requería un análisis detallado de la teoría de ecuaciones diferenciales introducida por Fuchs, la tercera versaba sobre las ecuaciones diferenciales no lineales de Briot y Bouquet, y la última estaba dedicada al estudio de las relaciones algebraicas conectando las funciones fuchsianas de Poincaré que tienen el mismo grupo de automorfismos.

\section{El efecto del concurso sobre Poincaré}

Cuando se publica el anuncio, Poincaré tiene solamente treinta y un años, pero ya es conocido dentro del mundo de las matemáticas por sus resultados sobre teoría cualitativa de ecuaciones diferenciales, topología y funciones fuchssianas o automorfas, entre otros. Además, los tres miembros de la comisión habían pensado en él como uno de los posibles concursantes, no solamente para atacar el problema de $n$ cuerpos, sino también al menos para el cuarto problema, ya que era Poincaré quien había introducido las funciones llamadas por él fuchsianas.

Como parece ser que a Poincaré lo costó un poco tomar la decisión de presentarse al concurso ${ }^{2}$, Mittag-Leffler decidió enviarle una carta el 13 de julio de $1887^{3}$, animándole a participar. Poincaré le contesta el 16 de julio, confirmándole su intención de hacerlo intentando resolver el primer problema. En la misiva, después de recordar Poincaré los resultados que ha encontrado para el problema restringido de tres cuerpos-donde se tienen dos puntos materiales, llamados primarios, girando uno alrededor del otro a lo largo de una circunferencia, y cuyo problema consiste en describir el movimiento de una tercera partícula de masa tan pequeña que

2 Para más detalles sobre Poincaré y el premio del Rey Óscar, véanse, por ejemplo, $[13,5,6,7]$

3 La referencia estándar para la correspondencia de Poincaré son los Archivos Henri Poincaré de la Universidad de Nancy 2, http:/www.univ-nancy2.fr / poincare/, y [24] en particular para la correspondencia entre Poincaré y Mittag-Leffler. 
no afecta al movimiento de los primarios-, le comenta a Mittag-Leffler que espera atacar el problema general no ya para resolverlo, empresa que le parece casi imposible, sino al menos para obtener unos resultados nuevos lo suficientemente relevantes para poder enviarlos al concurso.

A lo largo de dos años, Poincaré trabajó de manera persistente en el problema de tres cuerpos ${ }^{4}$. Al final de esos dos años, Poincaré había cimentado completamente su teoría cualitativa de ecuaciones diferenciales.

Así, fundamenta su teoría de invariantes integrales que especializa a sistemas hamiltonianos, donde el volumen es un invariante integral. Como aplicación, desarrolla su teorema recurrente, gracias al cual puede probar la estabilidad Poisson en sistemas conservando volumen confinados en regiones acotadas.

Profundiza sus estudios sobre órbitas periódicas y su estabilidad, obteniendo condiciones de linealización en un entorno de ellas cuando los exponentes característicos introducidos por él satisfacen una condición análoga a la descrita por el dominio de Poincaré en el entorno de un punto de equilibrio. Aquí Poincaré se dio cuenta de que podía obtener la solución completa cerca de órbitas periódicas gracias a la ausencia de pequeños divisores. Por otro lado, cuando éstos aparecían, daban lugar típicamente a la divergencia de los desarrollos.

Así, Poincaré reforzó el papel jugado por los exponentes característicos no triviales de órbitas periódicas no sólo para resultados de estabilidad, sino también para probar la inexistencia de integrales primeras uniformes para el problema restringido de tres cuerpos, lo cual generalizaba un resultado anterior, debido a Bruns, sobre la no integrabilidad algebraica.

Las integrales primeras son las constantes de movimiento, y la uniformidad significaba para Poincaré la dependencia analítica no sólo respecto a las variables del sistema, sino también respecto a los parámetros del sistema, y en particular en el problema restringido de los tres cuerpos respecto a la razón relativa entre las masas de los primarios. Después de obtener este resultado, Poincaré confirma que no es posible encontrar desarrollos en serie para la solución general del problema restringido de tres cuerpos que sean uniformemente convergentes, y se concentra en la demostración de la estabilidad del problema, para la que utiliza métodos geométricos similares a los de su teoría de ecuaciones diferenciales en el plano, es decir, de un carácter mucho más cuantitativo.

4 Como prueba de ello, se puede comparar la producción científica de Poincaré en los años 1888 y 1889 con la de los años anteriores y posteriores. La lista de publicaciones de Poincaré se puede encontrar también en http://www. univnancy2.fr/poincare/. 
Poincaré, creador de los métodos todavía modernos...

La existencia de exponentes característicos con partes reales no nulas da lugar a la aparición de órbitas periódicas de tipo silla, inestables por lo tanto, pero con separatrices asociadas, consistentes en las trayectorias que tienden hacia las órbitas periódicas para tiempos arbitrariamente positivos (superficie asintótica estable, en la terminología de Poincaré), o bien para tiempos arbitrariamente negativos (superficie asintótica inestable). Poincaré prueba que estas superficies asintóticas se cortan, y de aquí deduce, erróneamente, que tienen que coincidir, con lo que las trayectorias que se encuentran entre dos separatrices diferentes permanecen siempre allí y no se escapan al infinito, dando lugar así a un resultado de estabilidad del problema restringido de tres cuerpos, que es anunciado con cierto énfasis en la introducción de la memoria [35] que Poincaré presenta al concurso durante mayo de 1888 . Esta memoria, que representaba un avance espectacular en los métodos de la teoría de ecuaciones diferenciales, al combinar métodos cuantitativos con cualitativos y geométricos, ha sido considerada como el primer tratado sobre teoría cualitativa de ecuaciones diferenciales. Contenía tantas ideas nuevas que contribuyó significativamente a la idea general de que muy poca gente era capaz de entender los resultados de Poincarés. Sin embargo, la memoria que aún hoy en día continua siendo tan popular, no es la misma que la que Poincaré presentó al concurso.

\section{El concurso y el error}

Se presentaron doce memorias, de las cuales cinco, entre ellas la de Poincaré, versaban sobre el primer problema del movimiento de los $n$ cuerpos. Otra versaba sobre el tercer problema, y las seis restantes estaban dedicadas a otros problemas. En una primera selección, los miembros del comité, ayudados por Edvard Phragmén, uno de los editores d'Acta, destacaron a tres por encima del resto. Después de una lectura realizada en paralelo por Hermite en París, y por Weierstrass y Mittag-Leffler en Berlín, todos ellos coincidieron al cabo de un mes en que había una memoria que sobresalía por encima de las otras, que era la que se merecía claramente el premio. Aunque las memorias se presentaban anónimamente, no les fue difícil reconocer la autoría de Poincaré en la memoria ganadora.

5 Según Poincaré, no más de una docena; véase, por ejemplo, http://www.hpoincare.com/public/histoire/poincare1.htm. 
Ahora bien, una cosa era decidir cuál era la memoria ganadora y otra mucho más complicada justificarlo, y hacer además el trabajo de revisión necesario para que la memoria fuese publicada en Acta, tal como estaba establecido en las bases del concurso. Aquí Mittag-Leffler era el más interesado en obtener una versión final lo más clara posible, para facilitar su posterior difusión. Ahora bien, Mittag-Leffler conocía por anteriores lecturas de trabajos de Poincaré que sus escritos no se caracterizaban por una riqueza de detalles, por lo que sabía que este trabajo de revisión no sería nada fácil, aunque lo hiciese junto con Weierstrass. Además, había que tener en cuenta que la memoria presentada por Poincaré era muy larga (162 páginas), y contenía muchas ideas completamente nuevas.

Finalmente, después de haber identificado toda una serie de pasos oscuros, Mittag-Leffler se decidió a escribir a Poincaré en nombre de los tres miembros del tribunal—rompiendo así las reglas del concurso, en el cual supuestamente todas las memorias eran anónimas-, confirmándole que su memoria era una obra maestra, pero adjuntándole una lista de puntos que requerían una mayor explicación. Poincaré contestó enviando nueve Notas aclaratorias a modo de apéndice, que significaban 94 páginas más.

La comisión encontró otra memoria (debida a Paul Appell, compañero de estudios de Poincaré en Nancy) que era al menos merecedora de un reconocimiento, con lo que después de revisar ambas memorias, se encontró finalmente dispuesta a emitir sus resultados. En principio, era preceptivo también un informe (que, claro está, pasaría a la posterioridad), que si bien fue realizado por Hermite para la memoria de Appell, no ocurrió lo mismo con la memoria de Poincaré, ya que Weierstrass, quien era el encargado de hacerlo, se encontraba con frecuentes problemas de salud y no encontraba el tiempo y las fuerzas necesarias para llevarlo a cabo.

El 20 de enero de 1889, el día anterior al sexagésimo aniversario del Rey Óscar, el monarca aprobó oficialmente el resultado de la comisión que le fue presentado por Mittag-Leffler, consistente en la concesión del premio a Henri Poincaré, así como una mención honorable para Paul Appell. El resultado se publicó rápidamente en la prensa internacional, y Poincaré y Appell fueron considerados poco menos que héroes nacionales por la prensa francesa, ya que habían triunfado sobre la matemática alemana, que era la tradicionalmente considerada dominante. Posiblemente como consecuencia de este triunfo francés sobre Alemania, Poincaré y Appell fueron nombrados rápidamente caballeros de la Legión de honor.

Mittag-Leffler salió también muy reforzado en su calidad de organizador del concurso y editor jefe de Acta, y alimentaba la esperanza de que 
ambas memorias fuesen publicadas dentro del mismo 1889. De hecho, el volumen se acabó de imprimir durante el mes de noviembre de 1889, sin el informe de Weierstrass. Pero fue entonces cuando se encontró un importante error en la memoria de Poincaré que impidió la publicación del volumen ya impreso.

De hecho, la primera señal de que algo no funcionaba bien sucedió en julio de 1889 mientras Phragmén estaba revisando el artículo de Poincaré, en su calidad de editor de $A c t a^{6}$. Phragmén encontró algunos pasajes poco claros sobre la convergencia de los desarrollos de las series asintóticas en función del parámetro $\sqrt{\mu}$, donde $\mu$ es la razón relativa entre las masas de los cuerpos primarios en el problema restringido. Así se lo comunicó a Mittag-Leffler, quien escribió el 16 de julio una carta a Poincaré pidiéndole más detalles sobre este punto.

Poincaré no contesta a Mittag-Leffler hasta el uno de diciembre, comunicándole que el error detectado por Phragmén es grave:?

\begin{abstract}
Mon cher ami,
J'ai écrit à M. Phragmén pour lui parler d'une erreur que j'avais commise et il vous a sans doute communiqué ma lettre. Mais les conséquences de cette erreur sont plus graves que je ne l'avais cru d'abord. Il n'est pas vrai que les surfaces asymptotiques soient fermées, au moins dans le sens où je l'entendais d'abord. Ce qui est vrai, c'est que si je considère les deux parties de cette surface (que je croyais hier encore raccordées l'une à l'autre), se coupent suivant une infinité de courbes trajectoires asymptotiques (et de plus que leur distance est un infiniment petit d'ordre plus élevé que $\mu^{p}$ quelque grand que soit $p$ ). J'avais cru que toutes ces courbes asymptotiques après s'être éloignées d'une courbe fermée représentant une solution périodique, se rapprochaient ensuite asymptotiquement de la même courbe fermée. Ce qui est vrai, c'est qu'il y en a une infinité qui jouissent de cette propriété.

Je ne vous dissimulerai pas le chagrin que me cause cette découverte. Je ne sais d'abord si vous jugerez encore que les résultats qui subsistent, à savoir l'existence des solutions périodiques, celle des solutions asymptotiques, la théorie des exposants caractéristiques, la non-existence des intégrales uniformes et la divergence des séries de M. Lindstedt méritent la haute récompense que vous avez bien voulu m'accorder.
\end{abstract}

${ }^{6}$ Según Goroff [40], Phragmén fue uno de los concursantes, pero no parece haber ninguna otra constancia de que esto sea cierto.

${ }^{7}$ Poincaré a Mittag-Leffler, 1.12.1889. Para más detalles y comentarios sobre véase [5] y http://www.univ-nancy2.fr/poincare/chp/hpcochron.html. 
D'autre part, de grands remaniements vont devenir nécessaires et je ne sais si on n'a pas commencé à tirer le mémoire; j’ai télégraphié à M. Phragmén.

En tout cas je ne puis mieux faire que de confier mes perplexités à un ami aussi dévoué que vous l'avez toujours été. Je vous en écrirai plus long quand j'aurai vu un peu plus clair dans mes affaires.

Veuillez agréer, mon cher ami, avec mes bien sincères excuses, l'assurance de mon entier dévouement.

Poincaré

Esta carta es quizás el peor tipo de carta que puede recibir un editor de una revista y organizador de un premio, ya que la edición está ya acabada e incluso distribuida, y además puede acarrear serios perjuicios para el prestigio del premio y del comité el que se publique y se dé el premio a una memoria con un error esencial, sin contar con la repercusión que todo este asunto puede tener en la casa real sueca. Además, no queda claro en ella si el error tiene arreglo ni exactamente cuál es su abasto, así como cuándo se podrá arreglar.

Mittag-Leffler contesta a Poincaré el 4 de diciembre manifestándole su perplejidad al conocer estas noticias, pero añadiendo que está fuera de duda el que la memoria sea una obra maestra que será una referencia puntal en la mecánica celeste, confirmándole que en su opinión el premio le ha sido justamente otorgado. (Sin embargo, no es tan indulgente con Poincaré en otra carta enviada a Hermite dos días más tarde, y que se conserva en los archivos de l'Académie des Sciences, donde le comenta que el error cometido por Poincaré es tan grave que hay pocas páginas donde no se usan resultados que no sean falsos.)

Desafortunadamente, la carta de Poincaré ha llegado demasiado tarde, ya que la memoria original ha sido distribuida, y Mittag-Leffler se pone manos a la obra para intentar recuperar todos los ejemplares.

$\mathrm{Al}$ día siguiente, el 5 de diciembre, Mittag-Leffler le escribe otra carta a Poincaré, comunicándole que está en vías de recuperar prácticamente todos los ejemplares enviados, excepto los de Lindstedt y Gyldén que espera recuperar personalmente, y le sugiere que se haga cargo del costo de la edición del ejemplar malogrado (lo que Poincaré hará más adelante, teniendo que abonar más de 3.585 coronas, con lo que Poincaré de hecho perdió dinero en este concurso). Mittag-Leffler destruye todos los ejemplares, excepto al menos dos, que se han conservado en el Instituto Mittag-Leffler. (Uno de los cuales parece ser el que descubre Richard McGehee, durante una estancia post-doctoral al instituto [13].)

En la misma misiva, Mittag-Leffler le propone a Poincaré que escriba una nueva versión libre de error, pero sin hacer modificaciones muy profun- 
Poincaré, creador de los métodos todavía modernos...

das, simplemente añadiendo que en la introducción sólo se mencione que la versión publicada sólo es una modificación de la presentada, donde se inclur yen aclaraciones para algunos puntos, y se subsana un error encontrado.

Finalmente, a principios de enero de 1889 Poincaré envía a Phragmén una versión corregida de su memoria. En ella incorpora las notas dentro del cuerpo del texto, y realiza importantes avances, como la introducción de las órbitas doblemente asintóticas (más tarde llamadas homoclínicas por Poincaré en [39]) para corregir el error encontrado. Por culpa del retraso acumulado debido a la edición de otros trabajos, el volumen conteniendo la memoria de Poincaré tal como se la conoce hoy en día [36], junto con la memoria de Appell y el informe de Hermite sobre ésta última, no aparece hasta mediados de noviembre de 1890.

\section{Escisión de separatrices}

Tal como ya se ha dicho brevemente, el error de Poincaré estaba causado por su creencia de que las superficies asintóticas estable e inestable asociadas a una órbita periódica, al tener que cortarse por argumentos de conservación del volumen en sistemas hamiltonianos, tenían que coincidir.

Poincaré estudiaba el problema restringido de tres cuerpos, que es un sistema hamiltoniano con dos grados de libertad. Después de haber estudiado la existencia de órbitas periódicas de tipo silla, Poincaré presentó un modelo simple de Hamiltoniano a considerar para estudiar el tipos de movimiento que tenía lugar cerca de esas órbitas periódicas.

El modelo de Hamiltoniano escogido por Poincaré era de la forma $H(x, q ; y, p)$, donde $(x, q)$ juegan el papel de variables de posición, y $(y, p)$ son sus momentos asociados, y tenía la forma [36, pág. 220]:

$$
H(x, q ; y, p)=p+y^{2}-2 \mu \sin ^{2} \frac{x}{2}-\mu \varepsilon \cos q \sin x
$$

dependiendo de dos parámetros $\mu>0$ y $\varepsilon$, supuestamente pequeños, cuyas ecuaciones diferenciales asociadas son:

$$
\begin{aligned}
\dot{x}=\frac{\partial H}{\partial y}=2 y, & \dot{y}=-\frac{\partial H}{\partial x}=\mu \sin x+\mu \varepsilon \cos q \cos x \\
\dot{q}=\frac{\partial H}{\partial p}=1 & \dot{p}=-\frac{\partial H}{\partial q}=-\mu \varepsilon \sin q \sin x
\end{aligned}
$$


Para estudiar cualitativamente estas ecuaciones, Poincaré introdujo la aplicación tiempo $2 \pi$ o aplicación de Poincaré, que es el difeomorfismo del plano $(x, y)$ que a cada punto $\left(x_{0}, y_{0}\right)$ le asocia $P\left(x_{0}, y_{0}\right)=\left(x(2 \pi), y_{0}(2 \pi)\right)$, donde $(x(t), y(t))$ es la trayectoria del sistema con condiciones iniciales $x(0)=x_{0}, y(0)=y_{0}, q(0)=0, p(0)$. Tenemos así que la dinámica se reduce a la iteración de una aplicación 2-dimensional que además conserva área, como consecuencia de que las ecuaciones son hamiltonianas.

Si $\varepsilon=0$, el Hamiltoniano (1) se reduce a

$$
H(x, q ; y, p)=p+y^{2}-2 \mu \sin ^{2} \frac{x}{2},
$$

que es el hamiltoniano de un rotor lineal y un péndulo. Las ecuaciones asociadas poseen la solución $2 \pi$-periódica

$$
x=0, q=t, y=0, p=0,
$$

de energía $H=0$, que es de tipo silla, al ser sus exponentes característicos $\pm \sqrt{2 \mu}$. Las soluciones asintóticas (estable e inestable) asociadas a esta órbita periódica forman superficies dadas por las ecuaciones

$$
y=\frac{\partial S_{0}}{\partial x}, \quad p=\frac{\partial S_{0}}{\partial q}, \quad S_{0}=S_{0}(x, q)= \pm \sqrt{2 \mu} \cos \frac{x}{2},
$$

es decir

$$
y= \pm \sqrt{2 \mu} \sin \frac{x}{2}, \quad p=0
$$

y por tanto las superficies asintóticas para $e=0$ cierran una región cuya anchura es del orden de $\sqrt{\mu}$, y son por tanto doblemente asintóticas, es decir, asintóticas a la órbita periódica para $t \rightarrow \pm \infty$, y son comúnmente denominadas separatrices.

Si $\varepsilon \neq 0$, en la memoria presentada al concurso, Poincaré [35, pág. 143] creyó erróneamente que las superficies doblemente asintóticas, al tener que cortarse forzosamente por argumentos de conservación de área, tenían entonces que coincidir a todo orden, con lo que formaban una ba- 
Poincaré, creador de los métodos todavía modernos...

rrera que impedía el transporte a través de ellas, con lo que una trayectoria que en algún momento se encontraba entre ellas, tenía que continuar siempre dentro y no se podía escapar. Este argumento es el que le llevó a afirmar la estabilidad del problema restringido de tres cuerpos.

Sin embargo, en la memoria corregida [36, pág 222], Poincaré se dedica a buscar los puntos de intersección entre las superficies asintóticas, avanzándose en más de setenta años a lo que hoy en día recibe el nombre de método de Melnikov (véase, por ejemplo, [17]). Así, Poincaré buscó las superficies asintóticas en la forma

$$
y=\frac{\partial S}{\partial x}, \quad p=\frac{\partial S}{\partial q},
$$

donde $S(x, q ; \varepsilon, \mu)$ satisface la ecuación de Hamilton-Jacobi

$$
\frac{\partial S}{\partial q}+\left(\frac{\partial S}{\partial x}\right)^{2}=2 \mu \sin ^{2} \frac{x}{2}+\mu \varepsilon \cos q \sin x
$$

obtenida al sustituir la expresión de las superficies asintóticas (2) en la ecuación $H=0$, donde el hamiltoniano viene dado por (1). Al desarrollar $S$ en potencias de $\varepsilon$ :

$$
S=S_{0}+\varepsilon S_{1}+\varepsilon^{2} S_{2}+\cdots
$$

donde ya es conocido, se plantea completar en un paso más la expresión de $S_{1}$ mediante el cálculo de , que ha de cumplir la ecuación

$$
\frac{\partial S_{1}}{\partial q}+2 \sqrt{2 \mu} \sin \frac{x}{2} \frac{\partial S_{1}}{\partial x}=\mu \cos q \sin x
$$

Ahora bien, al resolver esta ecuación lineal para $S_{1}$, Poincaré llega a expresiones diferentes según se imponga que las superficies (2) sean asintóticamente inestables o estables. Para que coincidan, hace falta que el área que encierran sea nula. En la nueva memoria [36], Poincaré calcula esta área y observa que es proporcional a 


$$
\begin{gathered}
\varepsilon J+0\left(\varepsilon^{2}\right) \\
\text { donde } \quad J=2 \pi \sec \left(\frac{\pi}{2 \sqrt{2 \mu}}\right)=0\left(e^{-\pi / 2 \sqrt{2 \mu}}\right)
\end{gathered}
$$

Como $J$ es obviamente no nulo, Poincaré concluye que para $\varepsilon \neq 0$ pero pequeño, las variedades doblemente asintóticas no son cerradas. De hecho, comprueba que las variedades asintóticas se cortan en una infinidad de puntos, y con este resultado cierra prácticamente su memoria [36, pág. 223].

\section{Poincaré y el caos}

Su memoria sobre el problema de tres cuerpos fue la base sobre la cual se sustentó su famoso tratado sobre la mecánica celeste Les méthodes nouvelles de la mécanique céleste, [37, 38, 39]. A lo largo de este tratado, aparecen desarrollados los diversos temas de la memoria, frecuentemente con mucho más detalle.

En particular, Poincaré lleva a cabo el cálculo de las superficies asintóticas en el segundo tomo. Así, en [38, §225-§232] se dedica al cálculo analítico de la diferencia entre superficies asintóticas, obteniendo fórmulas análogas a (4). Poincaré, además, recalca el hecho de que la expresión obtenida en (4) es exponencialmente pequeña en el parámetro $\mu$, con lo que es un fenómeno que no se puede detectar simplemente desarrollando en serie respecto a dicho parámetro.

En el tercer tomo, que no aparece hasta 1899, es cuando Poincaré llama homoclínicas a las trayectorias doblemente asintóticas, y después de comprobar que típicamente aparecen una infinidad de ellas, hace el siguiente comentario [39, §397, pág. 389]:

Cuando se intenta representar la figura formada por estas dos curvas y sus intersecciones en número infinito donde cada una de ellas corresponde a una solución doblemente asintótica, estas intersecciones forman un tipo de enrejado, de tejido, de red de mallas infinitamente finas; cada una de estas curvas no puede volver a cortarse con ella misma, sino que tiene que plegarse sobre ella de una manera muy compleja para volver a cortar una infinidad de veces todas las mallas del entramado.

La complejidad de esta figura es tan chocante, que ni siquiera intento dibujarla: No hay nada más apropiado para darnos una idea de la complicación del problema de tres cuerpos y en general de todos los problemas de la Diná- 
Poincaré, creador de los métodos todavía modernos...

mica para los cuales no hay integral uniforme y donde las series de Bohlin son divergentes.

Hay quien afirma que esta es la primera descripción de lo que hoy en día se llama caos (véanse, por ejemplo, [13, págs. 40-41], así como [26]), porque además viene generado por la detección de trayectorias transversales doblemente asintóticas.

Sin embargo, como prueba clara del avance de Poincaré respecto a sus coetáneos, no hubo después de él una investigación sobre este tipo de comportamiento irregular (exceptuando, quizás, a Birkhoff). No fue hasta más de setenta años después, con la entrada en escena de los ordenadores, que apareció la posibilidad de la experimentación numérica de modelos que se aplicaban en diversos campos, con lo que comenzó una nueva ciencia multidisciplinar, denominada sistemas dinámicos por los matemáticos, dinámica no lineal por los físicos, y ciencia no lineal en general por el resto de disciplinas, donde aparecen frecuentemente fenómenos de tipo caótico.

En particular, fue Lorenz en [21] quien descubrió la existencia de dependencia sensible respecto a condiciones iniciales que se daba en un modelo simple de tres ecuaciones diferenciales, supuestamente pensado como modelo atmosférico. Este fenómeno se popularizó más adelante como efecto mariposa, cuando Lorenz impartió en 1972 una conferencia en l'American Association for the Advance of Science titulada Puede el aleteo de una mariposa en Brasil provocar un tornado en Texas? (véase [16]).

Poco después, Stephen Smale [42] introdujo un modelo matemático denominado la herradura de Smale donde se ponía de manifiesto la dependencia sensible respecto a las condiciones iniciales, junto con la existencia de una infinidad de órbitas periódicas de tipo silla así como de trayectorias densas en el retrato de fases. Más adelante Smale puso de manifiesto que la escisión de separatrices daba lugar en general a la existencia de herraduras de Smale, y por tanto a la existencia de movimiento caótico. Así, aunque Poincaré no pudo seguramente detectar esta dependencia sensible respecto a condiciones iniciales como movimiento caótico, sí detectó cómo calcular la escisión de separatrices, y que ésta origina un movimiento complicado, con lo que podemos considerar a Poincaré como el «abuelo» de la ciencia no lineal.

También al mismo tiempo, el método de escisión de separatrices desarrollado por Poincaré durante el breve tiempo de corrección del error en su memoria, fue redescubierto por Melnikov [22] y Arnold [3], dando lugar a lo que se conoce como el método de Poincaré-Melnikov-Arnold o, más brevemente, como el método de Melnikov. 
De hecho, Arnold desarrolla en [3] el método de la escisión de separatrices para detectar, en sistemas hamiltonianos con más de dos grados de libertad, la existencia de trayectorias que pueden escaparse una distancia arbitrariamente grande. Este fenómeno se conoce como la difusión de Arnold, y no podía darse en los hamiltonianos de dos grados de libertad estudiados por Poincaré por argumentos topológicos. Aquí cabe también destacar que los sistemas de más de dos grados de libertad son mucho más complicados por la aparición de los pequeños divisores en los desarrollos de las soluciones, un problema que no se resolvió hasta que apareció el teorema K.A.M. debido a Kolmogorov, Arnold y Moser [18, 2, 23].

Un fenómeno detectado por Poincaré, pero que no pudo tratarlo adecuadamente, fue el de la escisión de separatrices exponencialmente pequeñas respecto al parámetro de perturbación (véase [1] para unos comentarios recientes). Este fenómeno es muy importante porque también aparece en la difusión de Arnold, pero no fue estudiado hasta casi cien años más tarde.

Los primeros trabajos relacionados no aparecieron hasta 1984 cuando Neishtadt [25] pudo ofrecer cotas superiores para la escisión de separatrices en hamiltonianos de dos grados de libertad, y sobre todo cuando Lazutkin [19] introdujo su método basado en la parametrización de las superficies asintóticas para valores complejos de las variables, para la standard map definida sobre el plano.

Dichas cotas superiores para la escisión exponencialmente pequeña fueron obtenidas para familias de difeomorfismos por Fontich y Simó en [14]. Finalmente, la primera prueba sobre expresiones asintóticas para de la existencia de escisión de separatrices para sistemas como el estudiado por Poincaré fue debida a Delshams y Seara [11, 12], y las ideas de Lazutkin sobre la aplicación estándar fueron finalmente completadas por Gelfreich [15], quince años después de la aparición del artículo de Lazutkin.

Con respecto a sistemas con más de dos grados de libertad, no ha sido hasta muy recientemente que se han obtenido resultados sobre escisión de separatrices, debido a la dificultad añadida de que los pequeños divisores aparecen en los exponentes de las expresiones exponencialmente pequeñas. Así, los primeros resultados se deben a Simó [41] y Delshams et al. [8] para hamiltonianos de un grado de libertad perturbados casi periódicamente, y actualmente ya se dispone de resultados rigurosos para la escisión exponencialmente pequeña de separatrices en Hamiltonianos de $n$ grados de libertad $[20,9,10]$.

Aquí vale la pena recalcar que algunos de estos resultados, notablemente [20], han sido obtenidos gracias a una relectura de los trabajos de 
Poincaré, tanto de la memoria sobre los tres cuerpos [36], como de Les méthodes nouvelles de la mécanique céleste [38, 39], con lo que nos encontramos con que pasados más de cien años de los trabajos de Poincaré en ecuaciones diferenciales y la mecánica celeste, sus trabajos son aún fuente de nuevas ideas actuales. Esto sería muy difícil de encontrar en la obra de cualquier otro matemático.

\section{Referencias}

[1] Anosov, D.V. (2001) Poincaré and the problems of Oscar II. Istor.-Mat. Issled. (2), 6(41) 57-72, 387.

[2] Arnold, V.I. (1963) Proof of a theorem of A. N. Kolmogorov on the invariance of quasi-periodic motions under small perturbations. Russian Math. Surveys 18(5) 9-36.

[3] Arnold, V.I. (1964) Instability of dynamical systems with many degrees of freedom. Dokl. Akad. Nauk SSSR 156, 9-12.

[4] Arnold, V.I. (1988 segunda edición) Geometrical methods in the theory of ordinary differential equations. Ed: Springer-Verlag, New York. Serie: Grundlehren der Mathematischen Wissenschaften, vol. 250. ISBN 0-387-96649-8.

[5] Barrow-Green, J. (1997) Poincaré and the three body problem. Ed: American Mathematical Society, Providence, RI. Serie: History of Mathematics, vol 11. ISBN 0-8218-0367-0.

[6] Bottazzini, U. (2002) Poincaré, philosophe et mathématicien. Ed: Belin, Paris. Serie: Les Génies de la Science. ISBN 2-84245-055-8.

[7] Delshams, A (2004). La gran influència de la memòria de Poincaré sobre els problema dels tres cossos, 2004. Conferencia realizada el 29 de enero de 2004 en la FME de la UPC. Por aparecer.

[8] Delshams, A., Gelfreich, V., Jorba, À. y Seara, T. (1997) Exponentially small splitting of separatrices under fast quasiperiodic forcing. Comm. Math. Phys. 189, 35-71.

[9] Delshams, A., Gutiérrez, P. y Seara, T.M. (2004) Exponentially small splitting for whiskered tori in Hamiltonian systems: Flow-box coordinates and upper bounds. Discrete Contin. Dynam. Systems 11(4), 785-826.

[10] Delshams, A., Gutiérrez, P. y Seara, T.M. (2004) Exponentially small splitting for whiskered tori in Hamiltonian systems: Continuation of transverse homoclinic orbits. Discrete Contin. Dynam. Systems 11(4), 757-783.

[11] Delshams, A. y Seara, T. (1992) An asymptotic expression for the splitting of separatrices of the rapidly forced pendulum. Comm. Math. Phys. 150, 433-463.

[12] Delshams, A. Seara, T.M. (1997) Splitting of separatrices in Hamiltonian systems with one and a half degrees of freedom. Math. Phys. Electron. J. 3(4), 1-40. 
[13] Diacu, F. y Colmes, P. (1996) Celestial encounters. Ed: Princeton University Press, Princeton, NJ. ISBN 0-691-02743-9.

[14] Fontich, E. y Simó, C. (1990) The splitting of separatrices for analytic diffeomorphisms. Ergodic Theory Dynam. Systems 10, 295-318.

[15] V. G. Gelfreich (1999) A proof of the exponentially small transversality of the separatrices for the standard map. Comm. Math. Phys. 201(1), 155-216, 1999.

[16] Gleick, J. (1987) Chaos. Ed. Penguin Books, New York. ISBN 0-14009250-1.

[17] Guckenheimer, J. y Colmes, P. (1990) Nonlinear oscillations, dynamical systems, and bifurcations of vector fields. Ed: Springer-Verlag, New Cork. Serie: Applied Mathematical Sciences, volumen 42. ISBN 0-387-90819-6, xvi+459 págs.

[18] Kolmogorov, A. (1978, versión original en 1954) The general theory of dynamical systems and classical mechanics. En Foundations of Mechanics. Ed: Benjamin/Cummings, Reading, Mass. págs. 741-757 (apéndice).

[19] Lazutkin, V. (2003, versión original en ruso en 1984) Splitting of separatrices for the Chirikov standard map. Zap. Nauchn. Sem. S.-Peterburg. Otdel. Mat. Inst. Steklov. (POMI), 300, 25-55.

[20] Lochak, P., Marco, J.P. y Sauzin, D. (2003) On the splitting of invariant manifolds in multidimensional near-integrable Hamiltonian systems. Ed: Amer. Math. Soc. Serie: Mem. Amer. Math. Soc. Vol, 163.

[21] Lorenz, E.N. (1963) Deterministic nonperiodic flow. J. Atmospheric Sci., 20, 130-141.

[22] Melnikov, V. (1963) On the stability of the center for time periodic perturbations. Trans. Moscow Math. Soc. 12, 1-57.

[23] Moser, J. (1962) On invariant curves of area-preserving mappings of an annulus. Nachr. Akad. Wiss. Göttingen: Math.-Phys. Kl., 2, 1-20.

[24] Nabonnand, P. (1999) La correspondance entre Henri Poincaré et Gösta Mittag-Leffler. Birkhäuser Verlag, Basel. Serie: Publications des Archives Henri-Poincaré. ISBN 3-7643-5992-7.

[25] Neishtadt, A.I. (1984) The separation of motions in systems with rapidly rotating phase. J. Appl. Math. Mech. 48(2), 133-139.

[26] Parker, M.W. (1998) Did Poincaré really discover chaos? [Recensión del libro de F. Diacu and P. Colmes (1966) titulado Celestial Encounters, editado por Princeton Univ. Press, Princeton]. Stud. Hist. Philos. Sci. B Stud. Hist. Philos. Modern Phys. 29(4), 575-588.

[27] Poincaré, H. (1879) Sur les propriétés des fonctions définies par les équations aux différences partielles. Thèse présenté à la Faculté des sciences de Paris, 1er aoû 1879. Ed: Gauthier-Villars, Paris. (También en Oeuvres, tome I, XLIX-CXXXI.).

[28] Poincaré, H. (1881) Mémoire sur les courbes définies par une équation différentielle (I partie). J. Math. Pures Appl. 3(7), 375-422. (También en Oeuvres, tome I, 3-44.). 


\section{Poincaré, creador de los métodos todavía modernos...}

[29] Poincaré, H. (1882) Mémoire sur les courbes définies par une équation différentielle (II partie). J. Math. Pures Appl. 3(8), 251-296. (También en Oeuvres, tome I, 44-84.).

[30] Poincaré, H. (1884) Sur certaines solutions particuliéres du problème des trois corps. Bull. astronom. 1, 65-74. (También en Oeuvres, tome VII, 253-261.).

[31] Poincaré, H. (1884) Sur la convergence des séries trigonométriques. Bull. astronom. 1, 319-327. (También en Oeuvres, tome IV, 591-598.).

[32] Poincaré, H. (1885) Sur les courbes définies par les équations différentielles (III partie). J. Math. Pures Appl. 4(1), 167-244. (También en Oeuvres, tome I, 90-161.).

[33] Poincaré, H. (1886) Sur les courbes définies par les équations différentielles (IV partie). J. Math. Pures Appl. 4(2), 151-217. (También en Oeuvres, tome I, 167-222.).

[34] Poincaré, H. (1886) Sur un moyen d'augmenter la convergence des sé ries trigonométriques. Bull. astronom. 3, 521-528. (También en Oeuvres, tome IV, 599-606.).

[35] Poincaré, H. (1889). Sur le problème des trois corps et les èquations de la dynamique. Acta Math. 13, 1-255. (Corresponde a la memoria impresa, pero no publicada, que obtuvo el premio de S.M. el Rey Oscar II.)

[36] Poincaré, H. (1890) Sur le problème des trois corps et les èquations de la dynamique. Acta Math. 13, 1-270.

[37] Poincaré, H. (1887) Les méthodes nouvelles de la mécanique céleste. Tome I. Serie: Les Grands Classiques. Ed: Gauthier-Villars, París. ISBN 285367-093-7. (Solutions périodiques. Non-existence des intégrales uniformes. Solutions asymptotiques.)

[38] Poincaré, H. (1881) Les méthodes nouvelles de la mécanique céleste. Tome II. Serie: Les Grands Classiques. Ed: Gauthier-Villars, París. ISBN 2 85367-093-7. (Méthodes de MM. Newcomb, Gyldén, Lindstedt et Bohlin.)

[39] Poincaré, H. (1887) Les méthodes nouvelles de la mécanique céleste. Tome III. Serie Les Grands Classiques. Ed: Gauthier-Villars, París. ISBN 285367-093-7. (Invariant intégraux. Solutions périodiques du deuxième genre. Solutions doublement asymptotiques.)

[40] Poincaré, H. (1893) New methods of celestial mechanics. Vol. 3, tomo 13 de History of Modern Physics and Astronomy. Ed: American Institute of Physics, New York. ISBN 1-56396-116-4. (Integral invariants and asymptotic properties of certain solutions. Translated from the French, Revised reprint of the $1967 \mathrm{En}$ glish translation, With endnotes by G. A. Merman, Edited and with an introduction by Daniel L. Goroff.).

[41] Simó, C. (1994) Averaging under fast quasiperiodic forcing. En: Hamiltonian Mechanics: Integrability and Chaotic Behavior, editor J. Seimenis. Ed: Plenum, New York. ISBN 0-306-44808-4. (Ver también en NATO ASI Ser. B: Phys. 331, 13-34. Conferencia en Torun, Poland, 28 June-2 July 1993.).

[42] Smale, S. (1965) Diffeomorphisms with many periodic points. En Differential and Combinatorial Topology (A Symposium in Honor of Marston Morse). Ed: Princeton Univ. Press, Princeton, N.J. Páginas 63-80. 